\title{
Classification of Ultrasonic Signs Pre-processed by Fourier Transform through Artificial Neural Network Using the Echo Pulse Technique for the Identification of Defects in Welded Joints of Structural Steel
}

\author{
Renata Torres Melo Sotero ${ }^{1}$, Maria Cléa S. de Albuquerque ${ }^{1}$, Francisco G. de Paula ${ }^{1}$, Cláudia T. T. Farias ${ }^{1}$ and \\ Eduardo F. de Simas Filho ${ }^{2}$ \\ 1. Nondestructive Testing Research Group, Federal Institute of Bahia, Salvador 40301-015, BA, Brazil \\ 2. Electrical Engineering Program, Federal, University of Bahia, Salvador 40210-630, BA, Brazil
}

Received: April 29, 2015 / Accepted: May 18, 2015 / Published: May 25, 2015.

\begin{abstract}
It is due to the need to ensure the security and integrity of equipment, that the non-destructive tests have been increasingly used in the industrial sector. Among these, the ultrasonic pulse echo technique is the most used in industry, mainly for its simplicity and efficiency. With one transducer only, it is possible to emit the ultrasonic and receive the echo pulse. The ANNs (artificial neural networks) are artificial intelligence techniques that, when properly trained, align themselves to inspection tests becoming a powerful tool in the detection and fault identification. In this work, the echo pulse technique was used to detect discontinuities in welds, where ANNs were fed from the information obtained by digital signal processing techniques (Fourier transform), to identify and classify three distinct classes of defects. Results showed that with the combination of feature extraction by Fourier transformation and classification with neural networks, it is possible to obtain an automatic defect detection system in welded joints with average efficiency.
\end{abstract}

Key words: Non-destructive testing, digital signal processing, neural networks, echo pulse, ultrasound.

\section{Introduction}

Non-destructive tests are increasingly used in the detection of defects in welded joints, in order to guarantee the reliability and quality of equipment and industrial products. The ultrasonic testing has been used with this purpose due to the combination of simple execution and high efficiency in flaws identification [1, 2]. During the manufacturing process, discontinuities may arise and, if not detected and repaired, can cause great damage.

Among the various ultrasonic testing, the echo pulse method is widely used. One advantage of this

Corresponding author: Renata Torres Melo Sotero, undergraduate, research field: ultrasound testing with the echo pulse technique. E-mail: renata.sotero@hotmail.com. technique is its high sensitivity in the detection of small discontinuities and accurate detection of discontinuities in the field near the sonic beam [3]. In the pulse echo technique, the pulses emitted by the transducer in uniform time intervals [4] are introduced into the material. A single transducer emits and receives ultrasonic waves, enabling the location, dimensions and discontinuities present in the part.

The ANNs (artificial neural networks) are nonlinear mathematical tools inspired by the functionality of biological neurons, applied in pattern recognition, prediction and optimization of complex systems [5]. The ANNs are able to gain knowledge from the examples used for training and generalize to new entries that were not present in the training set [1]. 
Classification of Ultrasonic Signs Pre-processed by Fourier Transform through Artificial Neural Network 287 Using The Echo Pulse Technique for the Identification of Defects in Welded Joints of Structural Steel

In this work, the ANNs were used to help distinguish the ultrasonic signals from three distinct classes of defects in welded joints of previously produced structural steel. In order to assist in its best performance, the ANN underwent a digital processing using the Fourier transform, a much used tool in the processing of ultrasonic signals to observe different frequency components of signals [6]. This allows the representation of signals defined in time in the frequency domain [7].

\section{Materials and Methods}

The material used for the development of this work consisted of a common carbon steel plate welded by the welding processes at electric arc TIG and stick welding. Its dimensions are shown in Fig. 1. During welding, some defects such as lack of penetration, porosity and slag inclusion were purposely inserted randomly throughout the piece.

The methodology used in the development of the work consists of performing ultrasonic testing by echo pulse for signs of the regions of the body proof that were defective, first identified by a radiographic test. One hundred signals were acquired from each region: flawless, and those regions where we identified: porosity, lack of penetration and slags inclusions. The system used to acquire signals consisted of a pulse generator, an oscilloscope and a computer.

To acquire echo pulse test signals, two parallel lines were marked to the weld bead. The first line was drawn at a distance of $37.50 \mathrm{~mm}$ from the weld bead and the second with $43.30 \mathrm{~mm}$. These distances were chosen due to the interference of the field near the transducer related to shorter distances to the weld bead.

Then, using the MATLAB computing environment, ultrasonic signals were pre-processed (Fig. 2) by Fourier transform to feed the ANN training. There were approximately $70 \%$ of the signs used in each region for forming the neural network and $30 \%$ for the test.

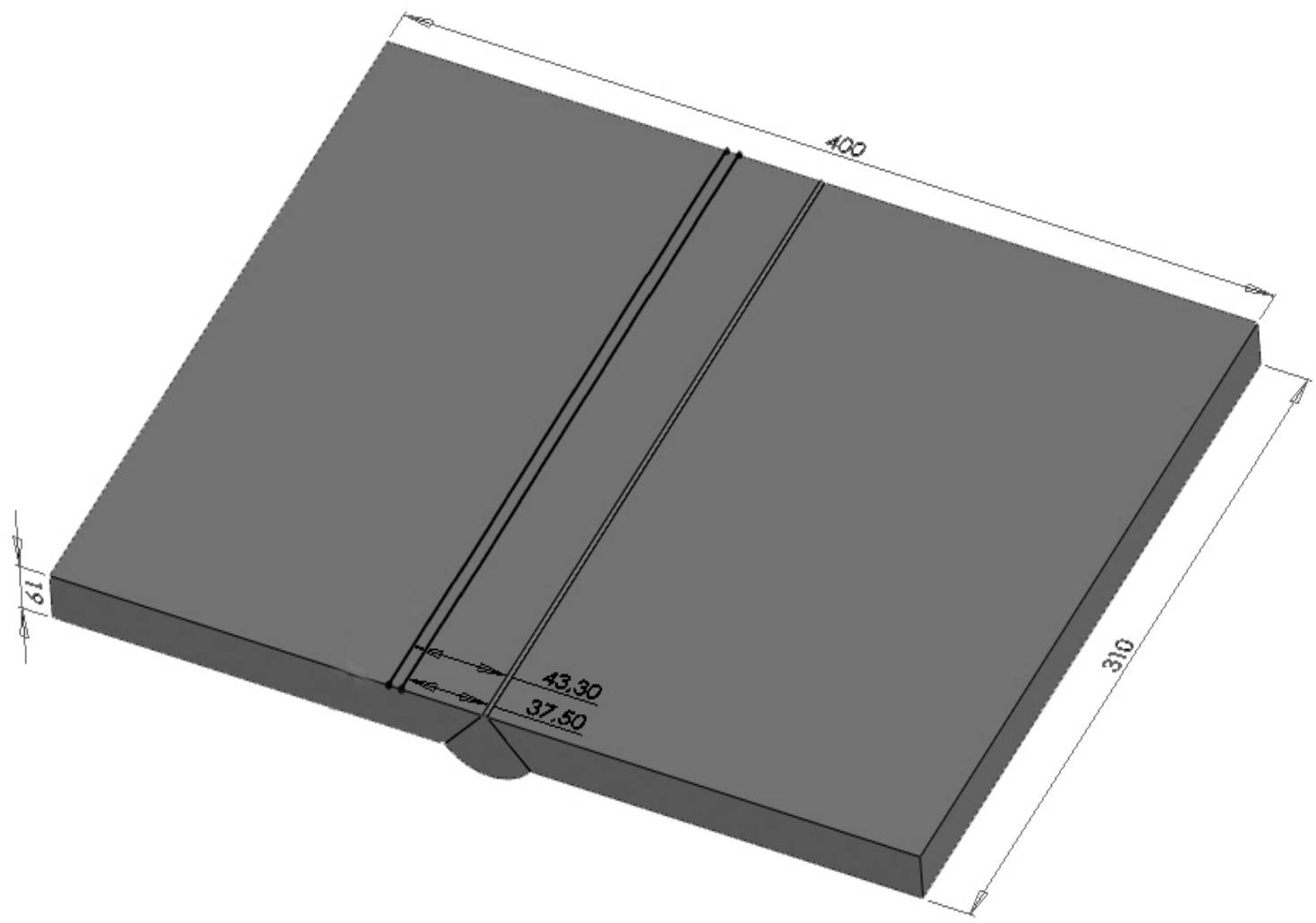

Fig. 1 Steel plate dimensions. 


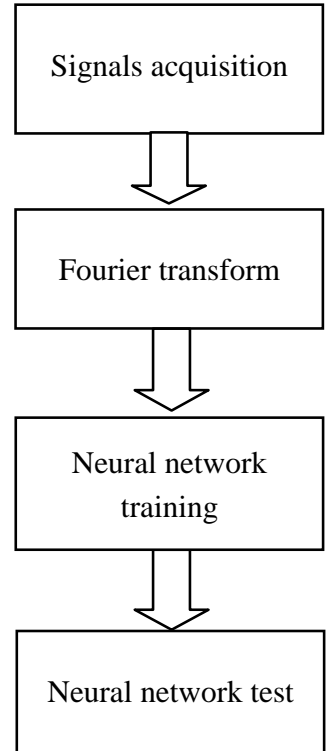

Fig. 2 Neural classifying flowchart [8].

\section{Experimental Results and Discussion}

In the acquisition step, 100 signals were obtained for each of the regions with and without weld defects: slag inclusion, lack of penetration and porosity. The Fourier transform was applied to the pre-processing of the
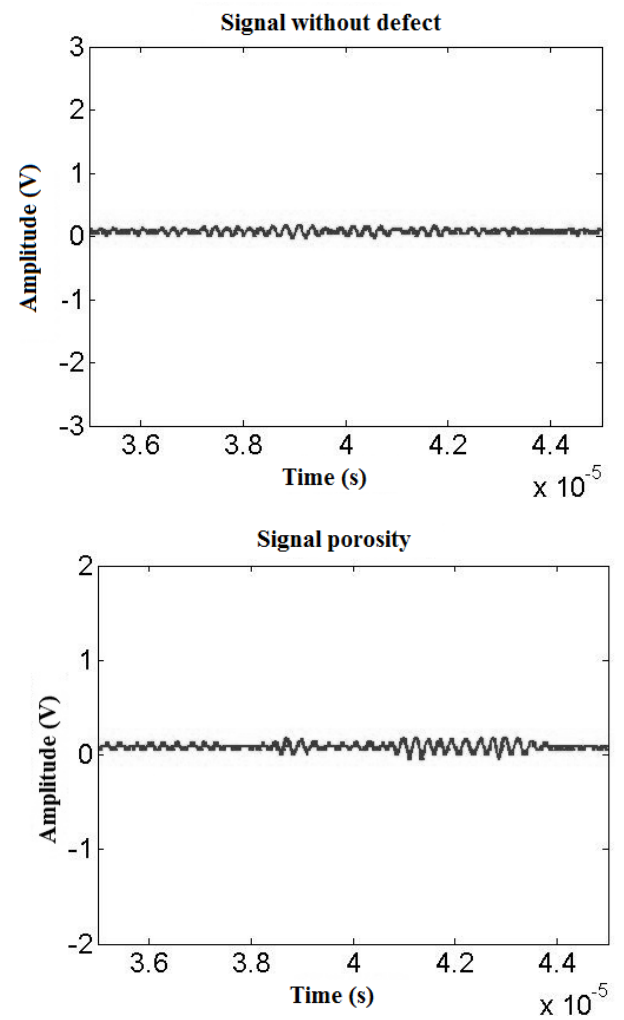

signal. Then, about 30 training neural networks were made and the best performance network was chosen for system operation.

Fig. 3 shows typical echo signals in the time domain into the four regions. The typical frequency domain signals are illustrated in Fig. 4.

To demonstrate the efficiency of the discriminator, Table 1 shows the results obtained by the neural classifier powered by the discrete Fourier transform. Based on the data, it can be seen that the highest efficiency was obtained with porosity class (83\%). The error was greater for the slag inclusion region (60\%). Thus, an average efficiency (geometric) of $73.5 \%$ was reached.

Ref. [8] compared the application of digital signal processing techniques, Fourier and Wavelet in identifying flaws in welded joints. Compared to their studies, we have seen that there is a similarity in the results obtained, thus proving that the technique is valid for detection of defects in welded joints of carbon steel plate.
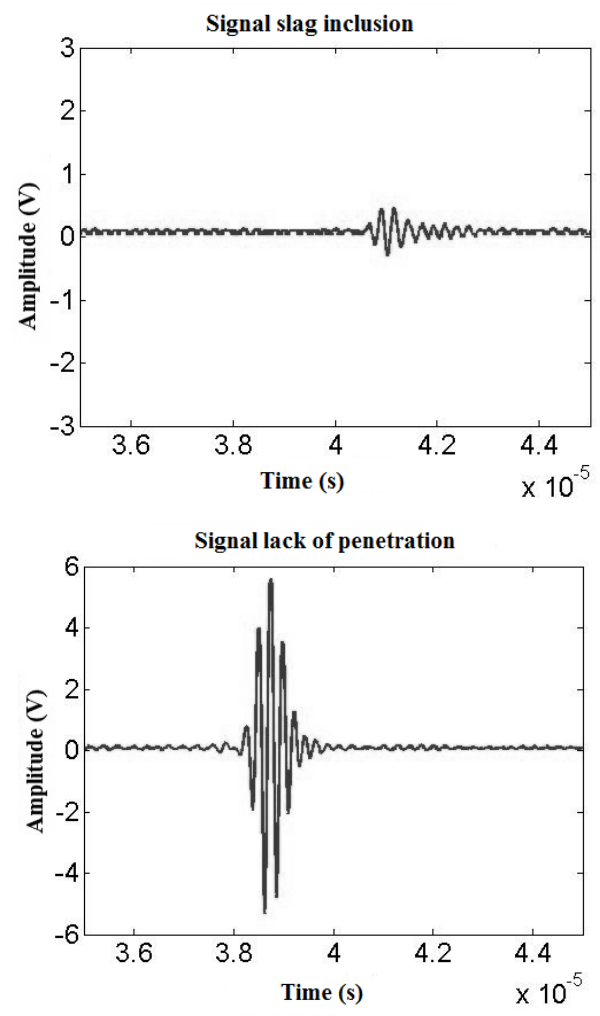

Fig. 3 Typical signs of echo pulse from faultless regions, with slag inclusion, porosity and lack of penetration. 
Classification of Ultrasonic Signs Pre-processed by Fourier Transform through Artificial Neural Network 289 Using The Echo Pulse Technique for the Identification of Defects in Welded Joints of Structural Steel
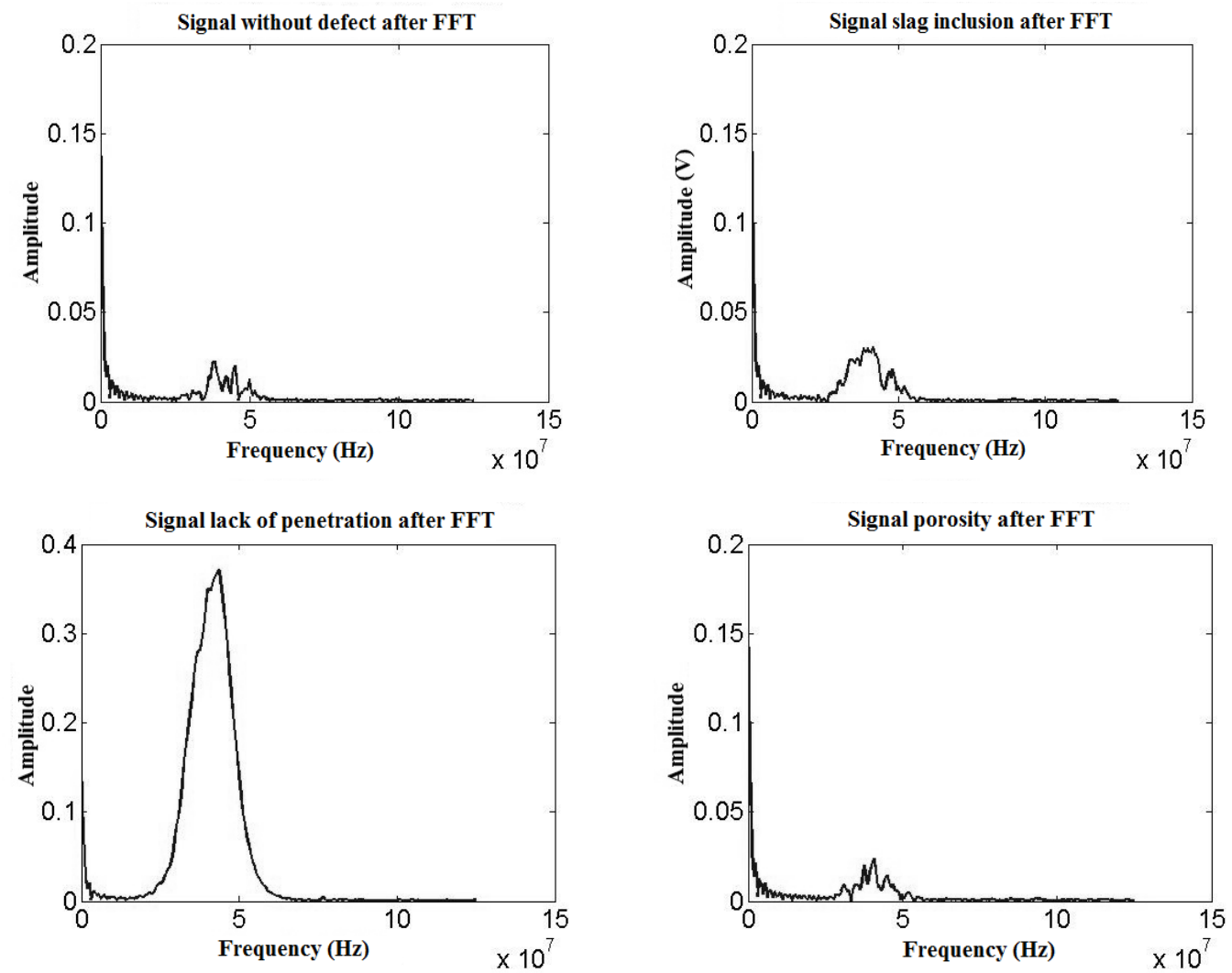

Fig. 4 Typical signals after pre-processing by Fourier transform obtained from the flawless regions and containing slag inclusion, porosity and lack of penetration.

Table 1 Matrix of confusion (in \%) obtained after the training of a neural classifier powered by the coefficients of the Fourier transform.

\begin{tabular}{lllll}
\hline Classes & Flawless & Lack of penetration & Slag inclusion & Porosity \\
\hline Flawless & 69 & 8 & 12 & 12 \\
Lack of penetration & 4 & 82 & 4 & 3 \\
Slag inclusion & 3 & 1 & 60 & 2 \\
Porosity & 24 & 9 & 24 & 83 \\
\hline
\end{tabular}

\section{Conclusions}

Non-destructive tests are important to ensure the reliability of equipment and structures. The ultrasonic test at the echo pulse configuration is widely used for presenting characteristics such as simplicity of execution and efficiency in detecting discontinuities. This work proposes an automatic system for identifying defects in welded joints, which combined pre-processing of ultrasonic signals by Fourier transform with a classifier based on an artificial neural network. For the classes of interest in the issue, an average efficiency of $73.4 \%$ has been reached.

\section{Acknowledgments}

The authors would like to thank FAPESB for the financial support during the realization of the present paper.

\section{References}

[1] Lee, W. J., Chang, W. V., Yang, W., and Kim, B. 2009. "Defects Detection Using a Smart Ultrasound Pulse-Echo Technique.” Polymer Composites 31 (6): 1105-12.

[2] Filho, E. F. S., Souza, Y. N., Lopes, J. L. S., Cláudia T. T., Farias, C. T. T., and Albuquerque, M. C. S. 2013. "Decision Support System for Ultrasound Inspection of Fiber Metal Laminates Using Statistical Signal 
290 Classification of Ultrasonic Signs Pre-processed by Fourier Transform through Artificial Neural Network Using The Echo Pulse Technique for the Identification of Defects in Welded Joints of Structural Steel

Processing and Neural Networks.” Journal Ultrasonics 53: 1104-11.

[3] Veiga, J. L. B. C., Alves, A. C., Silva, I. C., and Rebello, J. M. A. 2003. Automation of the Analysis of Ultrasonic Pulse-Echo Testing Using Artificial Neural Networks. Rio de Janeiro: PANDT. (in Portuguese)

[4] Santin, J. L. 2003. Ultrasonic: Technical and Application. 2nd edition. Curitiba: Printing and Publishing Unified. (in Portuguese)

[5] Brumatti, M. 2005. “Artificial Neural Networks.” Vitória, Espírito Santo, Brazil. (in Portuguese)

[6] Farias, C. T. T. 2006. "Use of Ultrasonic Lamb Waves in
Composite Materials Inspection Fibre-Metal Laminates.” Ph.D. thesis, COPPE/UFRJ. (in Portuguese)

[7] Kruger, S. E. 1996. "Echoes of Spectral Analysis and Signal Backscatter Ultrasonic Applied to Damage Detection for Hydrogen Steel.” M.Sc. thesis, Federal University of Rio de Janeiro. (in Portuguese)

[8] Souza, I. S. S., Albuquerque, M. C. S., Filho, E. F. S., and Farias, C. T. T. 2012. "Signal Processing Techniques for Ultrasound Automatic Identification of Flaws in Steel Welded Joints-A Comparative Analysis.” Presented at the 18th World Conference on Nondestructive Testing, Durban, South Africa. 